\title{
Uso do Percurso Cognitivo para avaliar a facilidade de aprendizado: um estudo de caso no software Geogebra para o perfil de alunos cegos
}

\author{
Patricia Blini Estivalete - PPGIE/UFRGS, pestivalete@gmail.com \\ Emerson Bianchini Estivalete - PPGEDU/PUCRS, emersonestivalete@gmail.com
}

Resumo: A interface de um sistema de geometria dinâmica deve guiar usuários pelas ações projetadas para executar uma tarefa. Assim, este artigo relata o uso do método de percurso cognitivo para avaliação da facilidade de aprendizado de tarefas do sistema Geogebra para o perfil de alunos cegos. As ações projetadas para tarefas de usuários precisam ser compatíveis com o modo de realizaram as tarefas. Para isso, o avaliador inspecionou a correspondência de recursos de audio com os recursos visuais durante o percurso das tarefas avaliadas. Como resultado dessa inspeção, será apresentado o estudo de caso que demonstra os problemas de interação encontrados relacionados às barreiras de acessibilidade.

Palavras-chave: Método de Percurso Cognitivo, Usabilidade, Acessibilidade, Signos Visuais

\section{Use of the Cognitive route to assess ease of learning: a case study in the Geogebra software for blind student profile}

\begin{abstract}
The interface of a dynamic geometry system must guide users through the actions designed to perform a task. Thus, this article reports the use of the cognitive pathway method to evaluate the ease of learning tasks of the Geogebra system for the profile of students blind. Actions designed for users' tasks need to be compatible with how they perform the tasks. For this, the evauator inspected the matching of audio resources with the visual resources during the course of the evaluated tasks. As a result of this inspection, the case study which shows the problems of interaction related to accessibility barriers, will be presented.
\end{abstract}

Keywords: Cognitive Path Method, Usability, Accessibility, Visual Signs

\section{Introdução}

Diante das demandas educacionais, os sistemas de geometria dinâmica permitem aos alunos construir conhecimentos, através da interação e manipulação de objetos geométricos, como: retas, circunferências e pontos em um ambiente computacional (ISOTANI e BRANDAO, 2004). A interação homem-computador (IHC) para Norman (1986), é um processo em que o usuário escolhe uma tarefa, percorre um plano de ações, aciona elementos da interface, percebe e interpreta o feedback do sistema e, ainda, avalia se seu objetivo foi cumprido. A interface de um sistema interativo engloba um conjunto de elementos dispostos em tela com a qual o usuário mantém contato fisico ou conceitual durante a interação (MORAN, 1981).

Para Barbosa e Silva (2010), o contato conceitual com a interface envolve a interpretação do usuário daquilo que ele percebe através do contato físico com os dispositivos de entrada e de saída durante o uso do sistema. 
Pesquisadores da área IHC evidenciam o quanto é importante avaliar uma interface de um sistema, pois os usuários esperam que seja fácil de usar, e, ainda, tenham uma experiência agradável e envolvente. Segundo Barbosa e Silva (2010), existem vários métodos para avaliar a qualidade de uso de um sistema propostos na área de IHC. Sendo que, cada método atende a um objetivo de avaliação, e indicando quando e onde os dados devem ser coletados, como eles devem ser interpretados e quais critérios de qualidade de uso como: usabilidade, experiência do usuário, acessibilidade ou comunicabilidade que sua avaliação vai atender. Ainda, leva em conta que os métodos de avaliação de IHC estão enquadrados em: métodos de inspeção, de observação e de investigação.

Desse modo, um conjunto de problemas relacionados com a facilidade e o esforço necessários para usuários aprenderem e utilizarem um sistema pode ser identificado a partir do uso da interface de um sistema de geometria interativa. Nielsen (1993) define o critério de usabilidade como um conjunto de fatores que qualificam quão bem uma pessoa pode interagir com um sistema interativo, endereçando a capacidade cognitiva, perceptiva e motora dos usuários empregada durante a interação.

Para a realização da avaliação por inspeção da interface, a área de IHC dispõe do percurso cognitivo, método que busca avaliar a facilidade de aprendizado. Em Barbosa e Silva (2010), os métodos de inspeção permitem ao avaliador explorar as tarefas dos usuários, com um determinado perfil, para identificar possíveis problemas que eles possam encontrar ao interagirem com o sistema e os modos de interação que o sistema oferece para ajudá-los a eliminarem as barreiras. A facilidade de aprendizado, que será o foco da pesquisa, refere-se segundo Barbosa e Silva (2010) ao tempo e esforço necessários para que o usuário aprenda a utilizar o sistema com determinado nível de competência e desempenho.

Isto posto, o presente artigo tem o objetivo de avaliar por meio de inspeção a facilidade de aprendizado da interface do sistema de geometria Geogebra considerando o perfil de alunos cegos.

\section{Método de Percurso Cognitivo}

No teste de percurso cognitivo, para realizar as observações desejadas, um ou mais avaliadores percorrem conjuntamente a interface inspecionando as ações projetadas para um usuário concluir cada tarefa utilizando o sistema. Durante as experiências de uso observadas, são registrados dados de acordo com os objetivos da avaliação.

Seguindo o método descrito por Barbosa e Silva (2006), a avaliação é composta por atividades como: preparação, coleta de dados, interpretação, consolidação e relato dos resultados. Na preparação, são definidos o perfil dos usuários, as tarefas para eles executarem no sistema via interface, a descrição das ações para realizar cada tarefa e a representação da interface.

$\mathrm{Na}$ coleta de dados e interpretação, percorre-se a interface seguindo as descrições de ações necessárias para realizar cada tarefa, para cada ação descrita, analisar se o usuário executaria corretamente as ações, guiadas pelas seguintes perguntas: "O usuário vai tentar atingir o efeito correto?", "O usuário vai notar que a ação correta esta disponível?", "O usuário vai associar a ação correta com o efeito que está tentando atingir?", "Se a ação for executada corretamente, o usuário vai perceber que está progredindo na direção de concluir a tarefa?". As perguntas do método auxiliam o avaliador detectar as ações que possuem problemas, impedindo que o 
usuário aprenda a interagir com a interface para realizar a tarefa. O avaliador, conhecendo o perfil dos usuários, deve relatar histórias de sucesso e insucesso ao responder as perguntas.

$\mathrm{Na}$ consolidação dos resultados, sintetizar os dados sobre: "o que o usuário precisa conhecer para realizar as tarefas", "o que o usuário deve aprender enquanto realiza as tarefas", "sugestões de correções para os problemas encontrados". No relato dos resultados, será apresentado um relatório com os problemas encontrados e sugestões de correção.

\section{Estudo de Caso - Avaliação do Geogebra usando o percurso cognitivo para usuários cegos}

O estudo de caso em que o método de percurso cognitivo foi aplicado objetivou identificar possíveis problemas relacionados a facilidade de aprendizado, ou seja, rupturas encontradas na conceitualização da tarefa e no feedback do sistema a cada ação percorrida na experiência de um aluno cego.

Em uma primeira etapa, foi realizado um estudo para conhecer como se processa os conceitos matemáticos em estudantes cegos. Os resultados demostraram que é necessário estimular a construção de conhecimentos por meio de recursos didáticos que possam ser percebidos pelos sentidos tátil-cinestésico, auditivo e olfativo. Para entender melhor as barreiras enfrentadas por esse perfil de usuário, o percurso cognitivo foi aplicado no software Geogebra avaliando as ações projetas para cada tarefa. Diante disso, o contexto que estava sendo investigado era o de facilidade de aprendizado para alunos cegos. Logo, uma questão que norteou a inspeção foi se as ações de cada tarefa eram compatíveis com o modelo conceitual e com o modo de realizarem as tarefas. Uma ação que era percebida na interface somente por uma representação visual e que não era percebida pelo sentido auditivo foram considerados uma hipótese de insucesso da interação. Ainda, durante a inspeção, foram aplicadas várias perguntas do método que auxiliaram o avaliador identificar as ações que impediam o usuário cego de aprender a interagir com o sistema para realizar uma tarefa. Com base nisso, foram detectados casos de insucesso para todas as ações de cada tarefa que foram inspecionadas, pois em nenhuma delas foram utilizados recursos que atendam os modos de interação via sinais sonoros. De acordo com o método, foi possível constatar que os problemas encontrados estão fortemente relacionados às barreiras de acessibilidade do sistema.

\subsection{Aplicação do Percurso Cognitivo e problemas encontrados}

Para a aplicação do método, foram identificados três tarefas para investigar possíveis problemas no percurso de interação com o perfil de alunos cegos. A seguir, apresentaremos os problemas encontrados, como resultado da aplicação do percurso cognitivo. 
Tarefa1: Construir uma reta selecionando dois pontos

Perfil de usuário: aluno cego

Sistema: Geogebra

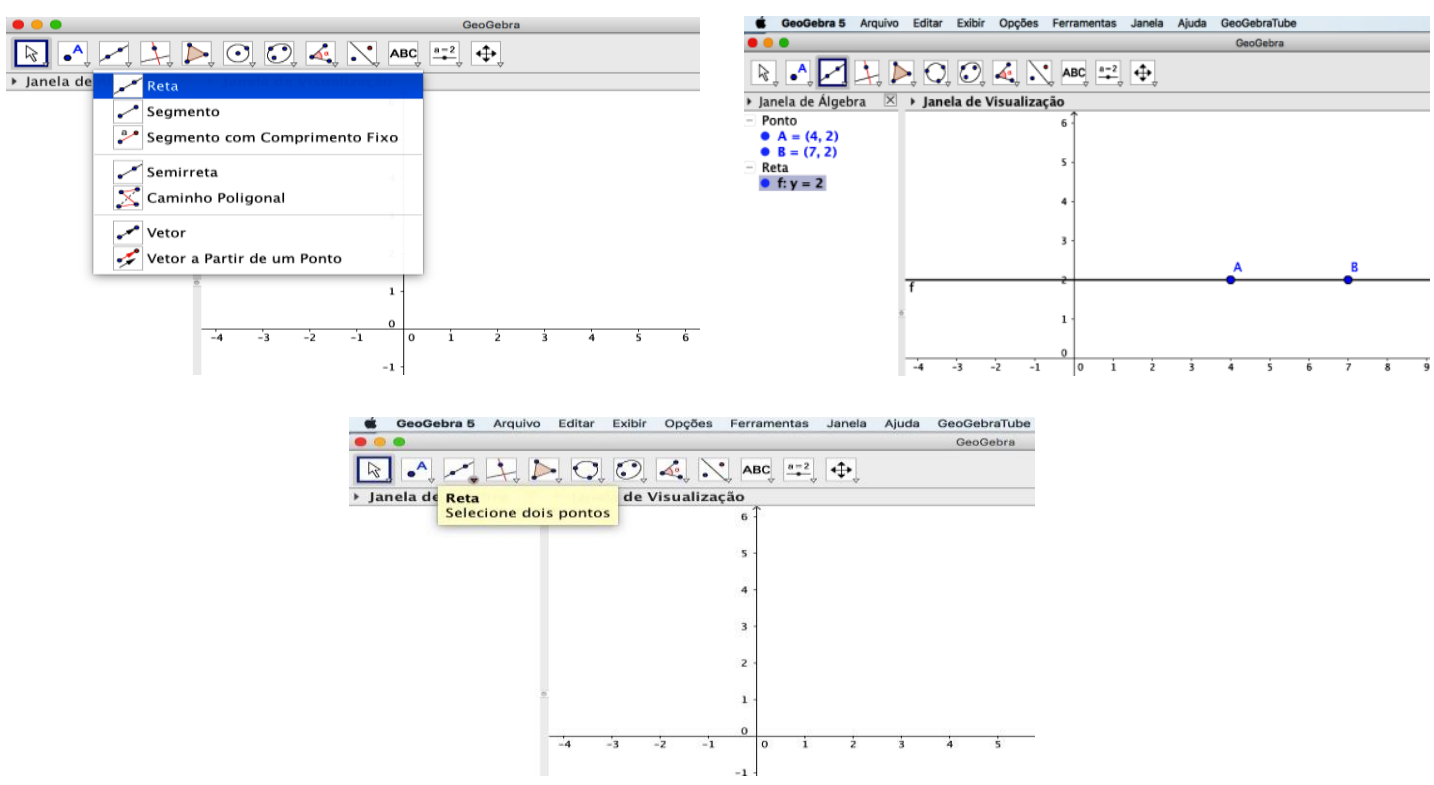

Figura 1 - Interface do Geogebra

Ações necessários para realizar a tarefa:

1. clicar na barra de ferramenta em "reta - selecione dois pontos"

2. clicar na opção "reta" no sub-menu

3. na janela de visualização selecionar um ponto A e um ponto B para formar a reta

Acão 1: clicar na barra de ferramenta em "reta - selecione dois pontos"

\section{P1: O usuário tentaria atingir o efeito correto?}

Não, o usuário não atinge o efeito de clicar com o mouse no signo visual da ação "reta selecione dois pontos" na barra de ferramentas, pois não existe um signo ${ }^{1}$ sonoro correspondente.

\section{P2: O usuário perceberia que a ação correta esta disponível?}

Não, o usuário não percebe a ação "reta - selecione dois pontos", pois não existe signo sonoro equivalente ao visual.

\section{P3: O usuário conseguiria associar a ação correta com o efeito que está tentando} atingir?

Não, o usuário não consegue associar o efeito de clicar com o mouse na ação correta: "reta - selecione dois pontos", porque não é emitido feedback sonoro.

P4: Se a ação correta for realizada, o usuário perceberia que está progredindo para concluir a tarefa?

Não, o usuário não percebe o sub-menu com as opções de construção de reta, pois não é emitido uma instrução sonora equivalente aos signos visuais.

\footnotetext{
${ }^{1}$ Um signo é a representação de um elemento que compõe a interface de um sistema interativo.
} 
Acão 2: clicar na opção "reta" no sub-menu

\section{P1: O usuário tentaria atingir o efeito correto?}

Não, o usuário não consegue clicar com o mouse no signo visual da ação "reta" no submenu, pois não existe um signo sonoro correspondente.

\section{P2: O usuário perceberia que a ação correta esta disponível?}

Não, o usuário não percebe a ação "reta", pois não existe signo sonoro equivalente ao visual.

P3: O usuário conseguiria associar a ação correta com o efeito que está tentando atingir?

Não, o usuário não consegue associar o efeito de clicar com o mouse na ação correta: "reta", porque não é emitido feedback sonoro.

P4: Se a ação correta for realizada, o usuário perceberia que está progredindo para concluir a tarefa?

Não, o usuário não percebe a janela de visualização para construir a reta a partir da seleção de dois pontos de referencia $\mathrm{A}$ e $\mathrm{B}$, pois não é emitido um signo sonoro equivalente aos signos visuais.

Ação 3: na janela de visualização selecionar um ponto A e um ponto B para formar a reta

\section{P1: O usuário tentaria atingir o efeito correto?}

Não, o usuário não atinge o efeito de clicar com o mouse na janela de visualização para selecionar dois pontos para formar a reta, pois não existe um signo sonoro correspondente.

\section{P2: O usuário perceberia que a ação correta esta disponível?}

Não, o usuário não percebe a ação de "selecionar um ponto A e um ponto B" para formar a reta, pois não existe signo sonoro equivalente ao visual.

P3: O usuário conseguiria associar a ação correta com o efeito que está tentando atingir?

Não, o usuário não consegue associar o efeito de clicar com o mouse na ação correta: "selecionar um ponto A e um ponto B" para formar a reta, porque não é emitido uma instrução sonora equivalente aos signos visuais.

P4: Se a ação correta for realizada, o usuário perceberia que está progredindo para concluir a tarefa?

Não, o usuário não percebe a construção da reta na janela de visualização, como também não percebe os pontos selecionados e a reta gerada na janela de álgebra, porque não é emitido um feedback sonoro.

Tarefa2: Construir um quadrilátero utilizando um polígono - selecionando todos os vértices

Perfil de usuário: aluno cego

Sistema: Geogebra 


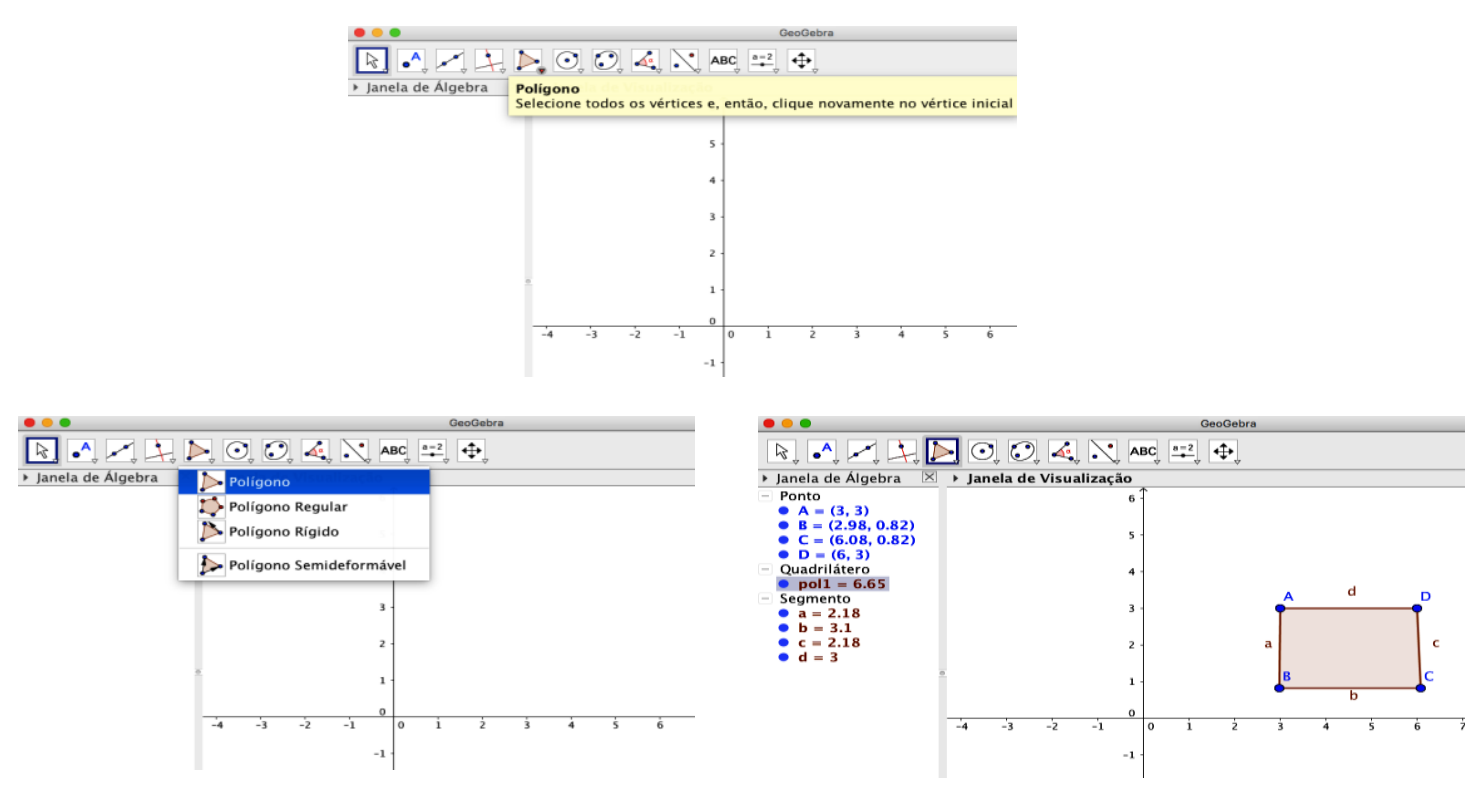

Figura 2 - Interface do Geogebra

Ações necessários para realizar a tarefa:

1. clicar na barra de ferramenta em "polígono - selecione todos os vértices e, então clique novamente no vértice inicial"

2. clicar na opção "polígono" no sub-menu

3. na janela de visualização selecionar um ponto $\mathrm{A}$, um ponto $\mathrm{B}$, um ponto $\mathrm{C}$, um $\mathrm{D}$ e por fim voltar a selecionar o ponto A

Ação 1: clicar na barra de ferramenta em "polígono - selecione todos os vértices e, então clique novamente no vértice inicial”

\section{P1: O usuário tentaria atingir o efeito correto?}

Não, o usuário não atinge o efeito de clicar com o mouse no signo visual da ação "polígono - selecione todos os vértices e, então clique novamente no vértice inicial" na barra de ferramentas, pois não existe um signo sonoro correspondente.

\section{P2: O usuário perceberia que a ação correta esta disponível?}

Não, o usuário não percebe a ação "polígono - selecione todos os vértices e, então clique novamente no vértice inicial", pois não existe signo sonoro equivalente ao visual.

\section{P3: O usuário conseguiria associar a ação correta com o efeito que está tentando atingir?}

Não, o usuário não consegue associar o efeito de clicar com o mouse na ação correta: "polígono - selecione todos os vértices e, então clique novamente no vértice inicial", porque não é emitido feedback sonoro.

P4: Se a ação correta for realizada, o usuário perceberia que está progredindo para concluir a tarefa?

Não, o usuário não percebe o sub-menu com as opções de construção de polígono, pois não é emitido uma instrução sonora equivalente aos signos visuais. 
Acão 2: clicar na opção "polígono" no sub-menu

\section{P1: O usuário tentaria atingir o efeito correto?}

Não, o usuário não consegue clicar com o mouse no signo visual da ação "polígono" no sub-menu, pois não existe um signo sonoro correspondente.

\section{P2: O usuário perceberia que a ação correta esta disponível?}

Não, o usuário não percebe a ação "polígono", pois não existe signo sonoro equivalente ao visual.

P3: O usuário conseguiria associar a ação correta com o efeito que está tentando atingir?

Não, o usuário não consegue associar o efeito de clicar com o mouse na ação correta: "polígono", porque não é emitido feedback sonoro.

P4: Se a ação correta for realizada, o usuário perceberia que está progredindo para concluir a tarefa?

Não, o usuário não percebe a janela de visualização para construir o polígono a partir da seleção de quatro pontos $(\mathrm{A}, \mathrm{B}, \mathrm{C}, \mathrm{D})$ e o retorno para o ponto $\mathrm{A}$ inicial, pois não é emitido um signo sonoro equivalente aos signos visuais.

Acão 3: na janela de visualização selecionar um ponto $\mathrm{A}$, um ponto $\mathrm{B}$, um ponto $\mathrm{C}$, um $\mathrm{D}$ e por fim voltar a selecionar o ponto A.

\section{P1: O usuário tentaria atingir o efeito correto?}

Não, o usuário não atinge o efeito de clicar com o mouse na janela de visualização para selecionar os pontos para formar o quadrilátero, pois não existe um signo sonoro correspondente.

\section{P2: O usuário perceberia que a ação correta esta disponível?}

Não, o usuário não percebe a ação de "selecionar um ponto $\mathrm{A}$, um ponto $\mathrm{B}$, um ponto $\mathrm{C}$, um ponto $\mathrm{D}$, e voltar a selecionar o ponto A" para formar o quadrilátero, pois não existe signo sonoro equivalente ao visual.

P3: O usuário conseguiria associar a ação correta com o efeito que está tentando atingir?

Não, o usuário não consegue associar o efeito de clicar com o mouse na ação correta: "selecionar um ponto $\mathrm{A}$, um ponto $\mathrm{B}$, um ponto $\mathrm{C}$, um ponto $\mathrm{D}$, e voltar a selecionar o ponto A" para formar o quadrilátero, porque não é emitido uma instrução sonora equivalente aos signos visuais.

P4: Se a ação correta for realizada, o usuário perceberia que está progredindo para concluir a tarefa?

Não, o usuário não percebe a construção do polígono na janela de visualização, como também não percebe os pontos selecionados e o quadrilátero gerado na janela de álgebra, porque não é emitido um feedback sonoro.

Tarefa3: Construir um círculo - selecionando o centro e, depois, um centro do círculo Perfil de usuário: aluno cego

Sistema: Geogebra 


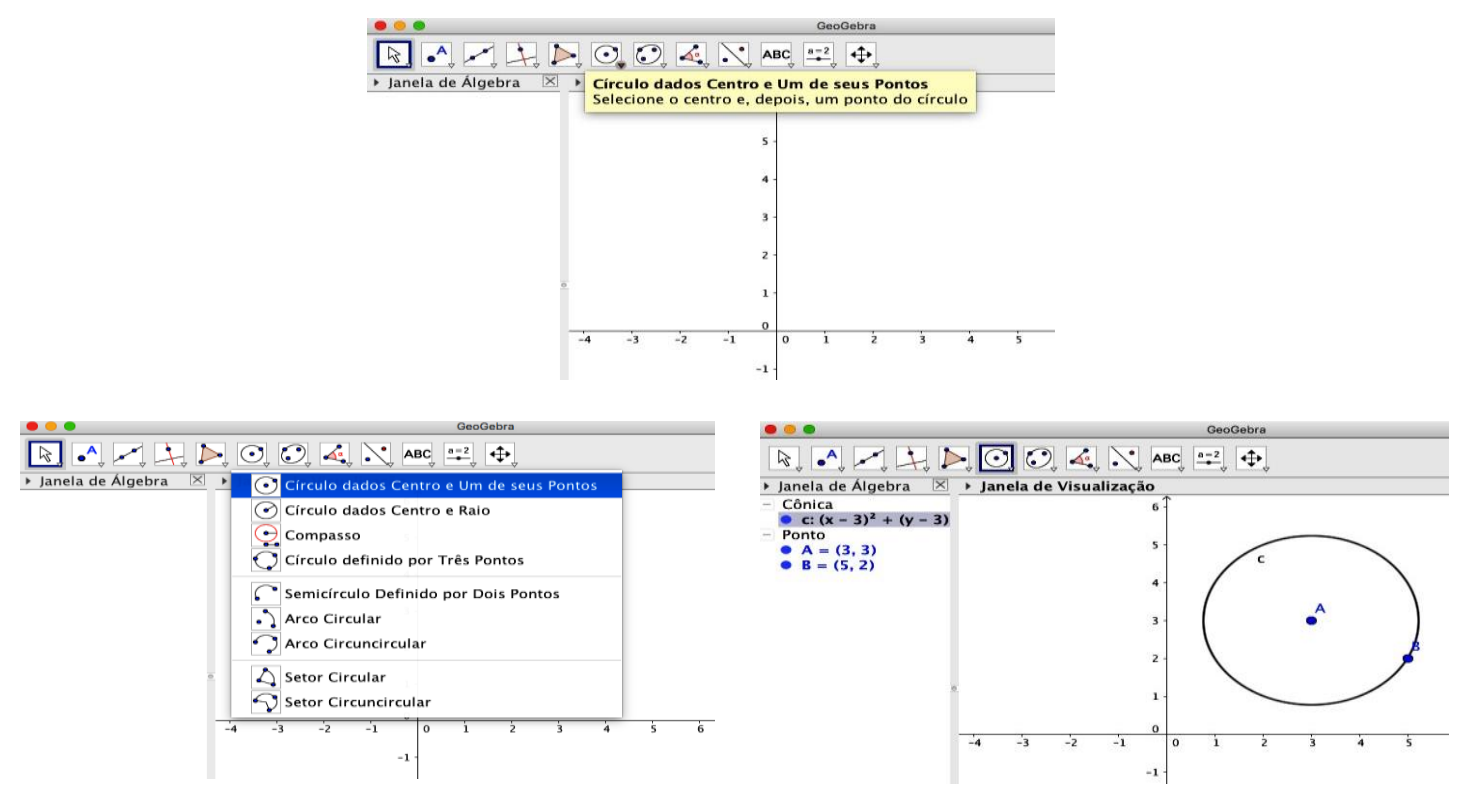

Figura 3 - Interface do Geogebra

Ações necessários para realizar a tarefa:

1. clicar na barra de ferramenta em "círculo dados centro e um de seus pontos selecione o centro e, depois, um ponto do círculo"

2. clicar na opção "círculo dados centro e um de seus pontos" no sub-menu

3. na janela de visualização selecionar um ponto A para o centro e um ponto B para um dos seus lados.

Acão 1: clicar na barra de ferramenta em "círculo dados centro e um de seus pontos selecione o centro e, depois, um ponto do círculo"

\section{P1: O usuário tentaria atingir o efeito correto?}

Não, o usuário não atinge o efeito de clicar com o mouse no signo visual da ação "círculo dados centro e um de seus pontos - selecione o centro e, depois, um ponto do círculo" na barra de ferramentas, pois não existe um signo sonoro correspondente.

\section{P2: O usuário perceberia que a ação correta esta disponível?}

Não, o usuário não percebe a ação "círculo dados centro e um de seus pontos - selecione o centro e, depois, um ponto do círculo", pois não existe signo sonoro equivalente ao visual.

P3: O usuário conseguiria associar a ação correta com o efeito que está tentando atingir?

Não, o usuário não consegue associar o efeito de clicar com o mouse na ação correta: "círculo dados centro e um de seus pontos - selecione o centro e, depois, um ponto do círculo", porque não é emitido feedback sonoro.

P4: Se a ação correta for realizada, o usuário perceberia que está progredindo para concluir a tarefa?

Não, o usuário não percebe o sub-menu com as opções de construção de círculo, pois não é emitido uma instrução sonora equivalente aos signos visuais. 
Ação 2: clicar na opção "círculo dados centro e um de seus pontos" no sub-menu P1: O usuário tentaria atingir o efeito correto?

Não, o usuário não consegue clicar com o mouse no signo visual da ação "círculo dados centro e um de seus pontos" no sub-menu, pois não existe um signo sonoro correspondente.

\section{P2: O usuário perceberia que a ação correta esta disponível?}

Não, o usuário não percebe a ação "círculo dados centro e um de seus pontos", pois não existe signo sonoro equivalente ao visual.

P3: O usuário conseguiria associar a ação correta com o efeito que está tentando atingir?

Não, o usuário não consegue associar o efeito de clicar com o mouse na ação correta: "círculo dados centro e um de seus pontos", porque não é emitido feedback sonoro.

P4: Se a ação correta for realizada, o usuário perceberia que está progredindo para concluir a tarefa?

Não, o usuário não percebe a janela de visualização para construir o círculo a partir da seleção de um ponto central A e um ponto B para qualquer ponto do círculo, pois não é emitido um signo sonoro equivalente aos signos visuais.

Acão 3: na janela de visualização selecionar um ponto A para o centro e um ponto B para um dos seus lados

\section{P1: O usuário tentaria atingir o efeito correto?}

Não, o usuário não atinge o efeito de clicar com o mouse na janela de visualização para selecionar os pontos para formar o círculo, pois não existe um signo sonoro correspondente.

\section{P2: O usuário perceberia que a ação correta esta disponível?}

Não, o usuário não percebe a ação de "selecionar um ponto A para o centro e um ponto B para um dos seus lados" para formar o círculo, pois não existe signo sonoro equivalente ao visual.

\section{P3: O usuário conseguiria associar a ação correta com o efeito que está tentando} atingir?

Não, o usuário não consegue associar o efeito de clicar com o mouse na ação correta: "selecionar um ponto A para o centro e um ponto B para um dos seus lados" para formar o círculo, porque não é emitido uma instrução sonora equivalente aos signos visuais.

P4: Se a ação correta for realizada, o usuário perceberia que está progredindo para concluir a tarefa?

Não, o usuário não percebe a construção do círculo na janela de visualização, como também não percebe os pontos selecionados do elemento cônico gerado na janela de álgebra, porque não é emitido um feedback sonoro.

\section{Considerações Finais}

Neste trabalho, o método de percurso cognitivo foi apresentado para avaliar a facilidade de aprendizado de tarefas do sistema Geogebra para o perfil de alunos cegos. A aplicação do método tinha a intenção de identificar problemas de interação relacionados às barreiras de acessibilidade. Por ser um contexto pouco explorado, tornase importante avaliar a acessibilidade das representações de uma interface, considerando 
o perfil do usuário, o seu modelo conceitual para realizar uma tarefa e o seu modo de interagir com os sistemas, buscando identificar problemas de uso no percurso de uma determinada tarefa.

Por inspecionar a facilidade de aprendizado de uma tarefa no sistema, o percurso cognitivo pode servir também como um método para avaliar a acessibilidade dos signos visuais por meio da equivalência sonora tendo como referência o usuário cego de maneira que possa perceber qual ação deve executar para realizar uma dada tarefa.

Por fim, este trabalho traz contribuições para as pesquisas relacionadas à aplicabilidade do percurso cognitivo e à acessibilidade de sistemas de geometria, considerando o perfil de alunos cegos.

\section{Referências Bibliográficas}

BARBOSA, S.D.J.; SILVA, B.S. da. Interação Humano- Computador. Elsevier Editora Ltda., 2010.

ISOTANI, S.; BRANDÃO, L. Ferramenta de avaliação automática no iGeom. In: Simpósio Brasileiro de Informática na Educação, 2004, Manaus. Anais. Manaus: Sociedade Brasileira de Computação.

MORAN, T. "The Command Language Grammars: a representation for the user interface of interactive computer systems". International Journal of Man-Machine Studies. 1981. Disponível em <http://www.sciencedirect.com/science/article/pii/ S0020737381800223> Acesso em : 10 Set. 20016.

NIELSEN, J. Usability Engineering. New York, NY: Academic Press, 1993. NORMAN, D. A. “Cognitive Engineering”. In: D.A. Norman e S.W. Drapear (eds.), User- Centered System Design. Hillsdale, NJ: Lawrence Erlbaum Associates, pp.31-61, 1986. 